\section{AD VALOREM}

Journal of Young Researchers

\section{ACCESo $\mathbf{0}$ ABIerto}

Para citaciones: Luna, J., Arrieta, J., Escobar, A. (2020). Metodología para el mejoramiento de la innovación organizacional en el sector logístico de Cartagena de Indias. Revista de jóvenes investigadores Ad Valorem, 3(2), 115-132

Editor: Bernardo Romero Torres. Universidad de Cartagena-Colombia.

Tipología IBN Publindex:

Artículo de investigación científica y tecnológica

\title{
Metodología para el mejoramiento de la innovación organizacional en el sector logístico de Cartagena de Indias
}

\author{
Jorge Armando Luna Amador ${ }^{1}$, July Arrieta Rodríguez ${ }^{2}$, Andrés Escobar Espinoza ${ }^{3}$
}

Universidad de Cartagena - Colombia

\section{RESUMEN}

Este artículo de investigación tiene como objetivo diseñar una metodología para el mejoramiento de la innovación organizacional en el sector logístico de la ciudad de Cartagena, Colombia. Se utilizó la metodología de estudio de casos múltiples, la cual parte del análisis de nueve ejes determinantes de la innovación organizacional (cultura, estrategia, planificación, personas, procesos, herramientas, productos/servicios, eficiencia y capitalización) en 5 empresas pertenecientes al sector. A partir de lo anterior, se diseñó una metodología que busca mejorar los niveles de innovación organizacional de las empresas objeto de estudio, basándose en el diseño estratégico, los procesos y los resultados.

Palabras clave: Innovación; Metodología; Organización; Sector Logístico.

\section{Methodology for the improvement of organizational innovation in the logistic sector}

\begin{abstract}
This research article has as objective to design a methodology for the improvement of organizational innovation in the Logistic Sector of Cartagena. The methodology used was the study of multiple cases, which starts with the analysis of nine organizational innovation axes (culture, strategy, planning, people, processes, tools, products/services, efficiency and capitalization) in five companies belonging to this sector. Therefore, a methodology was designed to seek for the improvement of organizational innovation, based on the strategic design, the processes and the results.
\end{abstract}

Key words: Innovation; Methodology; Logistic Sector; Organization.

Copyright: (C) 2020. Luna, J., Arrieta, J., Escobar, A. Este es un artículo de acceso abierto, distribuido bajo los términos de la licencia Creative Commons AtribuciónNoComercial-SinDerivados 4.0 la cual permite el uso sin restricciones, distribución y reproducción en cualquier medio, siempre y cuando que el original, el autor y la fuente sean acreditados.

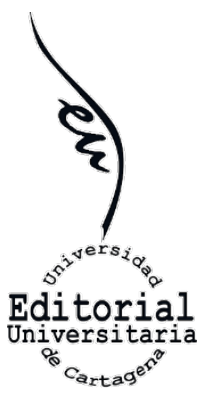

\footnotetext{
${ }^{1}$ MSc en Gestión de Organizaciones de la Universidad de Cartagena. Coordinador de Investigaciones en el Centro Logístico y de Negocios del Caribe. Correo: jlunaa@unicartagena.edu.co

${ }^{2}$ Magíster en Gestión de la Innovación de la Universidad Tecnológica de Bolívar. Ocean Import Coordinator en Kuehne + Nagel

${ }^{3}$ PhD en Economía de la Universita Cattolica del Sacro Cuore (Italia). Profesor en el Programa de Economía de la Universidad de Cartagena.
} 


\section{INTRODUCCIÓN}

La gestión de la innovación se constituye como una importante fuente de crecimiento sostenible y un factor determinante para alcanzar ventajas competitivas (Lam, 2010) que permitan a las empresas permanecer en un entorno organizacional complejo compuesto por mercados llenos de incertidumbre.

Partiendo de lo anterior, este artículo de investigación tiene como objetivo desarrollar una metodología para el mejoramiento de la innovación organizacional en el Sector Logístico para el Comercio Exterior en Cartagena, Bolívar; un sector que ha sido gran beneficiario de las políticas neoliberales de apertura económica implementadas en Colombia en los últimos años, tanto así, que de acuerdo a cifras del Sistema Estadístico de Comercio exterior (DIAN-SIEX), Cartagena ocupa el primer lugar de las aduanas colombianas por valor de US\$ 16.775 millones correspondientes al 60\% de mercancías tramitadas por esta aduana y comercializadas al exterior (Cámara de comercio de Cartagena, 2014).

La metodología utilizada para el desarrollo de esta investigación cuantitativa y explicativa, fue el estudio de casos múltiples, teniendo en cuenta que el análisis de la innovación organizacional es complejo, lo cual implica el estudio de múltiples participantes. Este estudio de caso se delimitó a cinco empresas del Sector Logística para el Comercio Exterior de la ciudad de Cartagena, las cuales, en primera instancia, fueron sometidas a encuestas estructuradas para determinar los niveles de innovación organizacional con base en el Modelo de Innovación Organizacional desarrollado por la Fundación COTEC (2013), una herramienta que ha tenido gran impacto en la comunidad académica y que ha servido como guía para el desarrollo de investigaciones como la que aquí se presenta.

En términos generales, para el desarrollo de este artículo se parte de una revisión rigurosa de los postulados de referentes teóricos que han contribuido a la construcción del concepto de innovación, profundizando en la innovación organizacional; seguidamente, se presenta la metodología utilizada para el determinar y analizar los niveles de innovación organizacional en las empresas objetivo de estudio para, posteriormente, desarrollar la metodología propuesta para mejorar los niveles de innovación organizacional en dichas empresas; finalmente, se muestran las conclusiones o consideraciones finales, producto de los resultados obtenidos durante el desarrollo de la investigación.

\section{Marco teórico}

Existen diferentes posturas y enfoques de académicos e investigadores sobre el concepto de innovación. Schumpeter (citado en Varela, 2001) plantea que en los sistemas capitalistas, las empresas se mantienen en un proceso evolutivo 
compuesto por nuevos bienes y servicios, nuevos métodos, mercados y estructuras organizacionales; de este proceso evolutivo radica la importancia de la innovación. La innovación según Schumpeter (citado en Sawhney, Walcott y Arroniz, 2006) no se trata de generar nuevo valor o cosas nuevas (invenciones), sino de crear valor para los clientes, por consiguiente, para la empresa.

Peter Drucker (1998) define la innovación como el esfuerzo que se hace por crear un cambio con un fin definido y con potencial comercial u organizacional para una empresa. Adicionalmente, indicó que, si bien tener ideas era fácil y tener buenas ideas complicado, lo realmente importante para la empresa es generar constantemente buenas ideas y materializarlas en productos, servicios o procesos. Por otro lado, Albornoz (2009) manifiesta que la innovación se encuentra en todas partes, pues se habla de innovación en la literatura científica y técnica, pero también en las ciencias sociales. Entonces la innovación ha pasado a ser un emblema de la sociedad que sirve para resolver muchos de los problemas que se presentan en un entorno inestable.

Actualmente, el fenómeno de la innovación se está estudiando desde una perspectiva multidimensional, la cual incluye varios tipos de innovación como son las innovaciones en producto, proceso, mercadotecnia y organización, siendo esta última el tipo de innovación a profundizar en este artículo (Dubrova, Ermolina y Esenin, 2019; Kita y Grossmanová, 2014). En este sentido, Yriyoguen (2013) también concibe la innovación como un fenómeno multidimensional, el cual tradicionalmente se ha limitado a la creación de nuevos productos, sin embargo, las organizaciones pueden innovar en diversas dimensiones organizacionales que le permitan satisfacer a sus clientes y posicionarse en el mercado (McDonough, Zack, Lin, \& Iris, 2008)

Según Lam (2010), cuando en la literatura académica se habla de la innovación organizacional se hace referencia a tres grandes áreas de estudio: a. La innovación en sí b. Los diferentes tipos de innovación en las organizaciones c. Los cambios en la estructura de la organización.

Garud, Tuertscher y Van de Ven (2013), por su parte, concentran el proceso innovador en cinco fases que son: ideas, resultados, personas, transacciones y conceptos. Afuach (1999), clasifica los procesos innovativos como radicales (nuevo conocimiento) e incrementales (mejora de un conocimiento existente). Hamel (2001), define la innovación organizacional como la capacidad de crear nuevas ideas de negocio o nuevas formas de diferenciar conceptos organizacionales. En este tipo de innovación se buscan nuevas formas organizacionales que modifiquen procesos internos y modifiquen las fronteras que separan a la empresa del mercado. 
La innovación organizacional tiene efectos en el precio (abono en los recursos), como tiene efectos en la calidad, (diferenciación de bienes y servicios). La Unión Europea (Comisión Europea, 1995), la describe como los mecanismos que generan interacciones internas empresariales y componen las redes con que la organización se comunica con su entorno. La RICYT (2001) -Red Iberoamericana de Ciencia y Tecnología- en revisión realizada al Manual de Bogotá, indica tres aspectos relevantes en la innovación organizacional que son los cambios en la gestión empresarial, los cambios en la estructura de la organización y los cambios en el direccionamiento estratégico. Finalmente, la OCDE (2005) define la innovación organizacional como la implementación de nuevos métodos en la práctica del negocio, los puestos de trabajo y en las interacciones empresaentorno.

El modelo de innovación organizacional de la Fundación COTEC (2013), el cual se toma como punto de partida para la metodología que aquí se diseña, plantea que la gestión de la innovación se fundamenta en cinco etapas interdependiente que son: focalizar, capacitarse, implantar, vigilar y aprender. Adicionalmente, consideran que las actitudes innovadoras en las empresas son: optar por la innovación, reconocerla como operación y valorar la innovación. Ahora bien, es importante mencionar que las empresas que componen los diferentes sectores económicos, tienen estructuras y procesos organizacionales particulares, por esta razón, es complejo estandarizar un modelo como el de la Fundación COTEC u otro para medir la innovación organizacional (Pertuz y Pérez, 2016). A partir de lo anterior, autores como Arraut (2010) en el sector petroquímico, Mauri y Canós (2004) en el sector logístico, Pertuz y Pérez (2016) en el sector de caficultores, entre otros; han diseñado y adaptado modelos específicos para medir la innovación organizacional en diferentes poblaciones empresariales.

El modelo de la Fundación COTEC, debido a su transversalidad, es uno de los más utilizados como base para la adaptación de modelos específicos de innovación organizacional. Este modelo ha servido de base para investigaciones de estudios de caso en el sector agrícola (Pertuz y Pérez, 2016), de servicios generales (D'alvano, 2011), hotelero (COTEC, 2007), industrias de las TIC (Bustamante, Dávila y Castañón, 2019), entre otros.

\section{Metodología}

Para el desarrollo de esta investigación, se utilizó el estudio de casos múltiples como herramienta metodológica, la cual es muy utilizada en la literatura de estudio de la organización (Bonache, 1999) y es la estrategia más adecuada cuando se busca responder el cómo y el porqué de un fenómeno, cuando el investigador tiene poco control sobre los hechos y acontecimientos, así como cuando se enfoca un fenómeno contemporáneo (Yin, 1994). Para Sosa (citado en Arraut, 2008), los estudios de casos pueden definirse como una investigación en 
profundidad sobre datos obtenidos en un periodo de tiempo determinado de una o más empresas, con el fin de generar un análisis del contexto y de los procesos implicados en el fenómeno objeto de estudio. En este sentido, este estudio de casos se presenta como una investigación empírica que estudia un fenómeno contemporáneo (innovación organizacional) dentro de su contexto real, en la que los límites entre el fenómeno y el contexto no son claramente visibles, y en la que se utilizan distintas fuentes de evidencia.

Los estudios de casos pueden ser simples cuando se refieren a una empresa o múltiples cuando participan dos o más empresas (Arraut, 2008).En este trabajo, se utiliza la metodología de estudio de casos múltiples explicativos debido a que se pretende entender las causas del fenómeno de estudio, partiendo del trabajo de campo para inducir hipótesis que expliquen los fenómenos organizativos. Para esto, se realizaron las mismas preguntas en cada caso, y se compararon las respuestas para llegar a conclusiones. Para definir el número de empresas de las unidades de análisis a incluirse en el diseño de múltiples casos, tomando como referencia que en esta metodología no opera el muestreo cuantitativo, se tuvo en cuenta la experiencia de autores como Arraut (2008) y Hernandez (2017), quienes establecen que en un estudio de este tipo se requieren como mínimo cuatro unidades de análisis (empresas). Además del concepto de Hernández (2017), quien afirma que entre mayor número de casos es posible un mayor entendimiento del problema, sin embargo, el número de casos más bien va a depender de los recursos económicos y el tiempo que disponga el investigador. Para la recolección de la información se utilizaron técnicas como cuestionario de encuestas, revisión documental, observación directa y colaboración de expertos en el sector, además se analizaron de cerca los procesos para la innovación que se realizan en el sector de Logística para comercio exterior. La información recogida a lo largo de la investigación, fue analizada de forma paralela al proceso de campo como se realiza en este tipo de estudios.

Las encuestas como instrumento utilizado para analizar el estado actual de la innovación organizacional en las empresas objeto de estudio, fue una adaptación de la herramienta desarrollada por la Fundación COTEC para la innovación Tecnológica, que valora las bases de un modelo empresarial de innovación en las PYMES (teniendo en cuenta que las empresas objeto de estudios están clasificadas como MIPYME según la Ley 590 del 2010 de la República de Colombia) y las obligaciones que sostienen la actitud innovadora de una empresa. El instrumento Autoevaluación de la PYME de la Fundación COTEC está conformado por 37 preguntas con opciones de respuestas Si, No. Adicionalmente, el investigador con la aprobación de un experto académico en innovación, en algunas preguntas agregó otras opciones de respuesta tales como: ¿Cuáles?, Detallar porcentaje frente a ingresos, ¿Los ha usado? ¿Cómo?, lo anterior con el fin de validar los datos recopilados inicialmente con las principales opciones de respuesta Si, No (Cotec, 2013). 
El modelo de la fundación COTEC, fue escogido para esta población de estudio (empresas del sector logístico para comercio exterior de la ciudad de Cartagena, Colombia) debido a su transversalidad (Arraut, 2010). Como se indicó en la revisión de la literatura, es un modelo que ha sido guía de estudios de casos simples y múltiples en empresas de diferentes sectores en diferentes países. Adicionalmente, se escoge como punto de partida este modelo, debido a su integralidad, ya que tiene en cuenta diversas variables de la actitud innovadora en la organización, tales como la cultura, la estrategia, la planificación, las personas, los procesos, herramientas, bienes y/o servicios, eficiencia y capitalización.

Con las 37 preguntas se busca analizar la dinámica de la innovación en las empresas objeto de estudio y las obligaciones que sostienen frente a la actitud innovadora PYME, cuyos sectores se clasifican en: a) optar por la innovación, b) Reconocerla como operación y c) valorizar la innovación. Asimismo, sobre cada uno de estos sectores se definen tres pilares o ejes para sustentar cada sector de innovación, es decir que se definen 9 ejes en total, los cuales son: Cultura, estrategia, planificación, personas/medios, procesos, herramientas, productos/servicios, eficiencia y capitalización. (COTEC, 2013).

La revisión documental, se constituye como una fuente de información primaria donde se obtienen datos relevantes de los procesos de la empresa. Aquí, se solicitó acceso al plan estratégico de las organizaciones, informes anuales, manuales procedimentales, documentos técnicos y comerciales de las propuestas de valor y documentación financiera. Adicionalmente, se hace una revisión de documentos públicos de las empresas, páginas web, estudios del sector logístico en Cartagena, investigaciones y literatura bibliográfica sobre innovación empresarial. Todo lo anterior, es útil para analizar que aspectos de innovación organizacional se encuentran debidamente documentados en las empresas objetos de estudio, y para dar sentidos a los resultados obtenidos en las encuestas.

La colaboración de expertos en el sector logístico, permitió contextualizar y analizar con mayor pertinencia los resultados obtenidos de las encuestas y la revisión documental. Adicionalmente, con su experiencia y conocimiento del sector logístico, se logró estructurar una metodología acorde a las necesidades de las empresas objeto de estudio, la cual podría proyectarse hacia las demás empresas del sector.

Finalmente, con la información recopilada y gracias a las distintas técnicas de recolección empleadas, se realizó un análisis riguroso que permitió el diseño de la metodología para mejorar la innovación organizacional en las empresas del Sector Logística de Cartagena Bolívar. Los métodos de recolección de información fueron aplicados en las cinco empresas objeto de estudio. 


\section{Resultados}

\subsection{Diagnóstico de innovación organizacional}

Al analizar los nueves ejes planteados en la metodología, se obtuvieron resultados que permiten determinar el estado actual de la innovación organizacional en las empresas objeto de estudio. Es imprescindible resaltar que para que las empresas cumplieran con los ejes de innovación organizacional, debían tener más de un $80 \%$ de cumplimiento de dicho eje. El estado de innovación organizacional ideal se alcanza cuando se cumplen todos los ejes. Cada uno de los nueve ejes, mide aspectos específicos de la innovación organizacional, se dice que una empresa alcanza el nivel de organización del eje cuando cumplen con la mayoría de las preguntas de dicho eje (COTEC, 2013).

Tabla 1. Descripción de los Ejes de Innovación Organizacional

\begin{tabular}{|l|l|}
\hline \multicolumn{1}{|c|}{ Eje } & \multicolumn{1}{|c|}{ Descripción } \\
\hline Cultura & $\begin{array}{l}\text { Divulgación de información de innovación a empleados, gestión } \\
\text { de procesos de innovación con proveedores, buen } \\
\text { relacionamiento con clientes, recompensa por aportar nuevas } \\
\text { ideas, la dirección de la empresa motiva a los empleados a } \\
\text { innovar, se tratan temas de innovación en reuniones de } \\
\text { directivos. }\end{array}$ \\
\hline Estrategia & $\begin{array}{l}\text { Asistencia a ferias y eventos de innovación, se constata la } \\
\text { existencia de objetivos específicos de innovación dentro de los } \\
\text { planes organizacionales, se conoce los programas de gobierno } \\
\text { de apoyo a la innovación empresarial, se hace publicidad de } \\
\text { nuevos bienes o servicios, poseen alianzas estratégicas con } \\
\text { entidades de capacitación y universidades, }\end{array}$ \\
\hline Planificación & $\begin{array}{l}\text { Se elaboran al interior de la empresa planes y proyectos de } \\
\text { innovación, se establece un presupuesto específico de la } \\
\text { empresa para actividades de innovación. }\end{array}$ \\
\hline Personas/medios & Capacitación periódica al personal sobre temas de innovación \\
\hline Procesos & $\begin{array}{l}\text { Procesos formalizados para participar en programas } \\
\text { gubernamentales de fomento a la innovación empresarial, } \\
\text { poseer un departamento u área especialmente dedicada a } \\
\text { procedimientos de innovación. }\end{array}$ \\
\hline Herramientas disponibles en la empresa para medir la \\
innovación, para obtención de información de los clientes y \\
métodos de innovación.
\end{tabular}

Fuente: Elaboración propia con base en COTEC 2013 
Para el caso de la empresa PTOM, se puede observar que alcanza un nivel ideal (100\%) de innovación organizacional en los ejes de cultura, estrategia, planificación, personas/medios, procesos, productos/servicios y capitalización. Por otro lado, en los ejes de herramientas y eficiencia, no se alcanza el nivel ideal de innovación organizacional.

Figura 1. Variables de innovación en PTOM

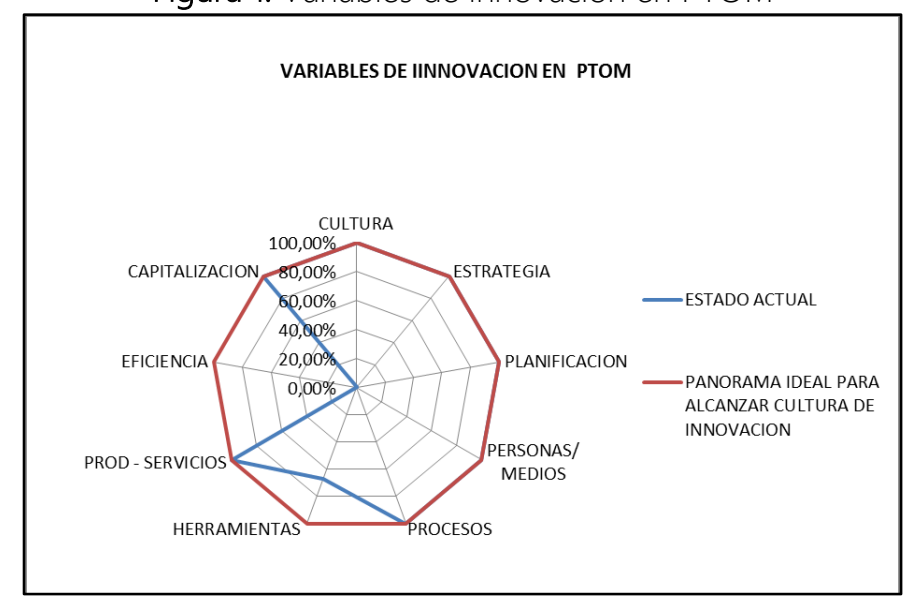

Fuente: Elaboración propia basado en COTEC 2013.

La empresa PTOS, presenta niveles ideales de innovación organizacional en los ejes de cultura, planificación, personas/medios, herramientas, productos/servicios y eficiencia. Mientas que no alcanzó los niveles requeridos en los ejes de estrategias procesos y capitalización.

Figura 2. Variables de innovación en PTOS

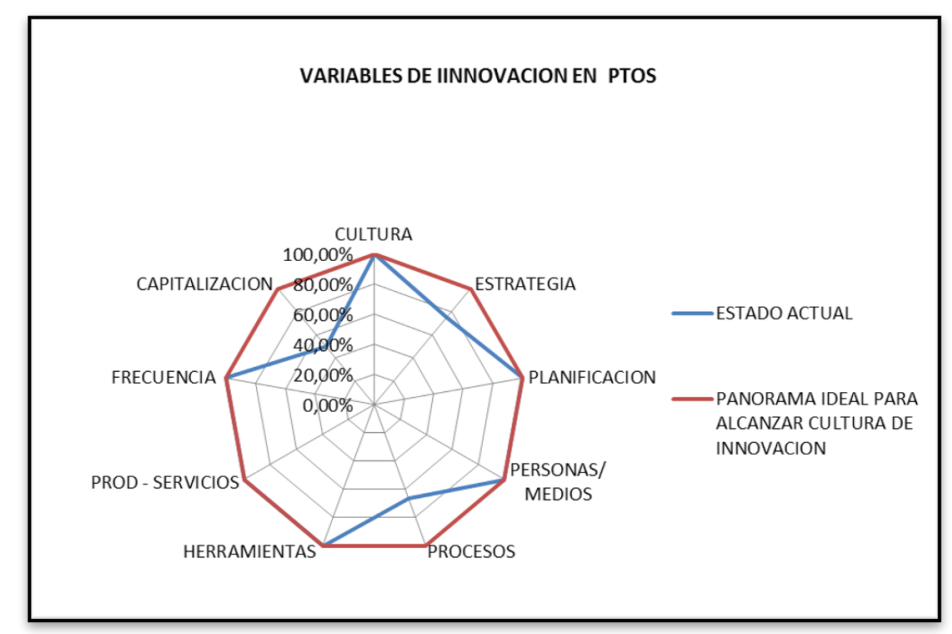

Fuente: Elaboración propia basado en COTEC 2013.

La empresa MROL, alcanzó los niveles de innovación organizacional en los ejes de cultura, estrategia, planificación, personas/medios y capitalización. Sin 
embargo, en los ejes de procesos, herramientas, productos/servicios y eficiencia no se alcanzó el nivel requerido.

Figura 3. Variables de innovación en MROL

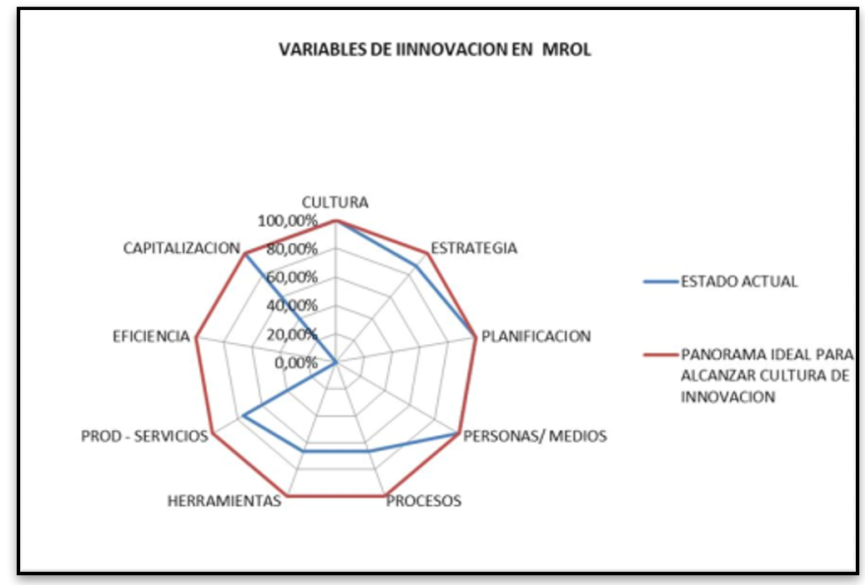

Fuente: Elaboración propia basado en COTEC 2013.

Por su parte, la empresa SERP se destacó con niveles aceptables de innovación organizacional en los ejes de cultura, planificación, personas/medios y capitalización. Por otro lado, en los ejes de estrategia, procesos, herramienta, productos/servicios y eficiencia no alcanzaron el nivel requerido.

Figura 4. Variables de innovación en SERP

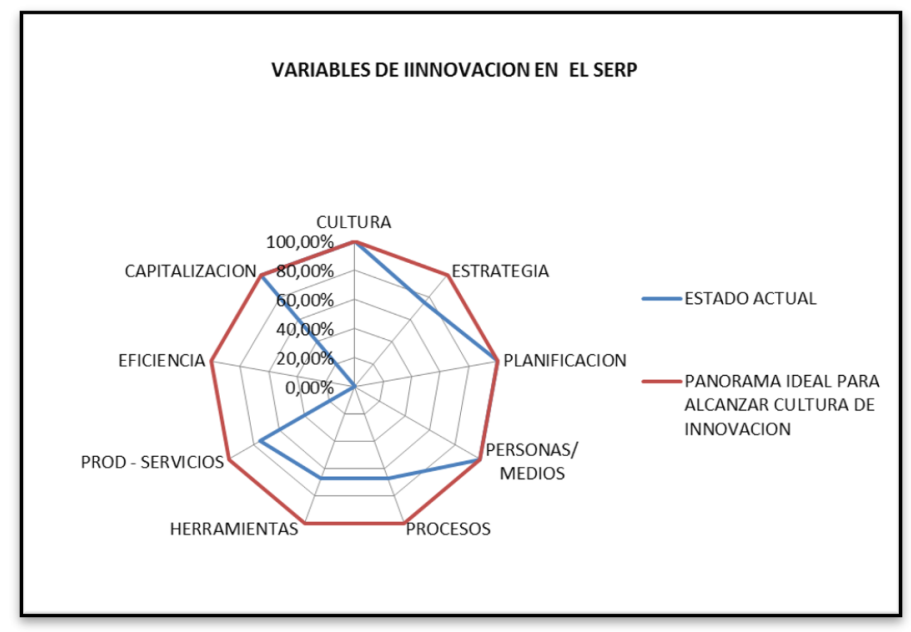

Fuente: Elaboración propia basado en COTEC 2013

Finalmente, la empresa INTG alcanzó niveles aceptables de innovación organizacional en los ejes de planificación, procesos, eficiencia y capitalización; mientras que en los ejes de cultura, estrategia, personas/medios, herramientas y productos/servicios no se alcanzaron el nivel de $80 \%$ requerido. 
Figura 5. Variables de innovación en INTG

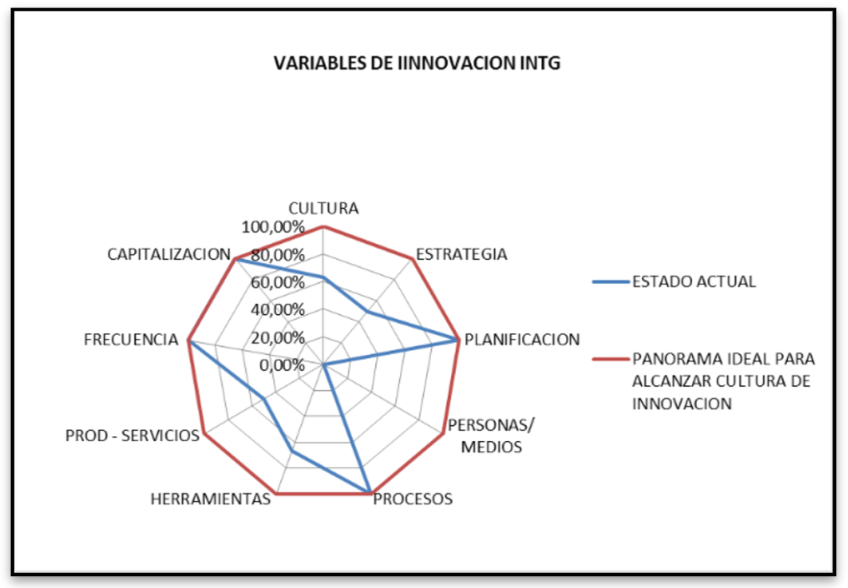

Fuente: Elaboración propia basado en COTEC 2013

En términos generales y, como se puede observar en la Tabla 2, ninguna de las empresas analizadas alcanza un estado de innovación organizacional ideal. PTOM fue la empresa que alcanzó el mayor grado de cumplimiento de los ejes de la innovación empresarial (cultura, estrategia, planificación, personas/medios, proceso, producto/ servicio y capitalización), mientras que INTG fue la que obtuvo el menor grado de cumplimiento con solo cuatro ejes (planificación, procesos, eficiencia y capitalización).

Respecto a los ejes, el que obtuvo el mayor grado de cumplimiento fue el de planificación que se cumplió en cada una de las empresas, mientras que el eje relacionado con las herramientas fue el de menor grado de cumplimiento al alcanzarse los resultados en solamente una empresa. Lo anterior muestra que en las empresas se están aprobando presupuestos para desarrollar proyectos de innovación de innovación, sin embargo, las empresas no cuentan con herramientas físicas y tecnológicas suficientes, lo cual puede implicar una barrera en la materialización de los proyectos de innovación planificados.

Tabla 2. Grado de cumplimiento de innovación organizacional de las empresas objeto

\begin{tabular}{cccccc}
\hline EJES/EMPRESAS & PTOM & PTOS & MROL & SERP & INTG \\
\hline Cultura & SI & SI & SI & SI & NO \\
Estrategia & SI & NO & SI & NO & NO \\
Planificación & SI & SI & SI & SI & SI \\
Personas/Medios & SI & SI & SI & SI & NO \\
Procesos & SI & NO & NO & NO & SI \\
Herramientas & NO & SI & NO & NO & NO \\
Producto/ Servicio & SI & SI & NO & NO & NO \\
Eficiencia & NO & SI & NO & NO & SI \\
Capitalización & SI & NO & SI & SI & SI \\
\hline
\end{tabular}

Fuente: Elaboración propia. 


\subsection{Propuesta metodológica para el mejoramiento de la innovación organizacional}

Con base en el diagnóstico estratégico, se obtuvieron unos resultados importantes que son imprescindibles al momento de diseñar la propuesta metodológica para el mejoramiento de la innovación organizacional en la población objeto de estudio. Algunos de estos resultados se describen a continuación:

- La planificación es fundamental para el mejoramiento de la innovación organizacional, pues para crear una cultura innovadora en una empresa, es necesario que haya un plan o direccionamiento estratégico estipulado, con unos objetivos y estrategias que permitan alcanzar la innovación organizacional. La importancia de la planificación, se demuestra al observar que el 100\% de las empresas analizadas cumplen con este eje, como base para alcanzar el estado ideal de innovación organizacional. En este orden de ideas, la metodología propuesta debe partir de una planificación o direccionamiento estratégico enfocado en la innovación organizacional.

- Es importante asegurar la disponibilidad de recursos, procesos formalizados y herramientas para llevar a cabo los proyectos de innovación organizacional. En las empresas analizadas en la fase de diagnóstico, se observó que tenían algunos recursos y procesos de innovación, sin embargo, al no contar con las herramientas necesarias, eran procesos que no se podían materializar efectivamente. Esto, pone de manifiesto que en la metodología propuesta, se confiera importancia a las herramientas dentro de los procesos de innovación.

- Cuatro de las cinco empresas analizadas, están trabajando en pro del alcance de una cultura innovadora, lo cual coloca la cultura como el segundo eje más desarrollado en las empresas después de la planificación. Lo anterior, puede indicar que dentro de los resultados a alcanzar con la propuesta, debe tenerse en cuenta la importancia de alcanzar una cultura innovadora en la organización.

Es importante mencionar, que el sector logístico para comercio exterior abarca diferentes eslabones de la cadena logística (agencias de aduanas, terminales portuarias, agentes marítimos, navieras, transportadores terrestres, empresas de servicio de pilotos prácticos, empresas de servicios de remolcadores, depósitos aduaneros, zonas francas, agentes de carga internacional, empresas de servicio de buceo), por lo cual es complejo diseñar un propuesta totalmente ajustada a todas las empresas pertenecientes a los distintos eslabones de la cadena de valor de este sector. Todas y cada una de las empresas logísticas, cuentan con distintos procesos operativos para la prestación de su servicio, sin embargo, existe un común denominador, pues todas y cada una de ellas están constituidas en pro al 
buen funcionamiento del comercio exterior y de las actividades logísticas derivadas este.

La presente metodológica busca ser de aplicabilidad en las empresas del sector, con la posibilidad de adaptarse de acuerdo a las necesidades y estructura organizativa de cada empresa. A continuación, se presenta la ruta de la metodología, teniendo en cuenta el diagnóstico hecho a las empresas objeto de estudio y, teniendo en cuenta las propuestas metodológicas de referentes teóricos como Arraut (2008) y Acevedo y Linares (2008), quienes indican la importancia del diseño estratégico, los procesos y resultados.

A partir de lo anterior, se plantea esta propuesta metodológica que parte de la variable Diseño Estratégico, debido a que la innovación en las empresas estudiadas es un tema que debería concebirse desde el nivel estratégico y coordinadamente hacerlo fluir a los demás niveles jerárquicos de las organizaciones, propiciando una inclusión de la innovación en la empresa como parte de su estrategia (Acevedo y Linares, 2008), luego se pasa a la variable Proceso, esta fase se refiere a los procesos, herramientas y actividades requeridas para impulsar la innovación en la empresa, por último abarca la variable Resultados para identificar los resultados de toda la gestión de innovación desarrollada en las variables anteriores. A continuación, se describirán a profundidad cada una de las variables anteriores y se diseñarán algunos indicadores de gestión respecto a las mismas para su respectivo control, monitoreo y seguimiento:

Tabla 3. Variables de metodología para el mejoramiento de la innovación empresarial en la fase Diseño Estratégico.

\begin{tabular}{|c|l|l|}
\hline FASE & VARIABLES & \multicolumn{1}{c|}{ DESCRIPCIÓN } \\
\hline \multirow{5}{*}{$\begin{array}{c}\text { Diseño } \\
\text { estratégico }\end{array}$} & Alta dirección & $\begin{array}{l}\text { Reúne inicialmente la cabeza de la organización (Socios), } \\
\text { inclusión de la innovación en los valores corporativos, misión, } \\
\text { visión y objetivos de la organización. En la parte de objetivos } \\
\text { hay que tener en cuenta que los objetivos trazados deben ser } \\
\text { alcanzables y coherentes con la realidad. }\end{array}$ \\
\cline { 2 - 5 } & Recursos. & $\begin{array}{l}\text { Asignación de un presupuesto económico para la gestión de } \\
\text { la innovación, autorización para involucrar todo el talento } \\
\text { humano que conforma la organización en sus diferentes } \\
\text { departamentos, autorización de espacios y tiempo para } \\
\text { realizar actividades de innovación. En este sentido la } \\
\text { innovación no se contempla como una pérdida de tiempo. }\end{array}$ \\
\cline { 2 - 5 } & $\begin{array}{l}\text { Búsqueda e } \\
\text { identificación del } \\
\text { recurso humano } \\
\text { para la } \\
\text { innovación }\end{array}$ & $\begin{array}{l}\text { Identificación del líder y responsable de la gestión de la } \\
\text { innovación, quien debe inspirar al resto de la organización, } \\
\text { búsqueda, participación y evaluación de grupos de } \\
\text { innovación por departamentos cuyos grupos para la gestión } \\
\text { de innovación están compuestos por diferentes profesionales } \\
\text { de diferentes áreas, lo ideal es la búsqueda de diversidad de } \\
\text { profesiones que hagan parte de la organización. }\end{array}$ \\
\hline
\end{tabular}

Fuente: Elaboración propia. 
Tabla 4. Medición de Variables de metodología para el mejoramiento de la innovación empresarial en la fase Diseño Estratégico.

\begin{tabular}{|c|c|c|}
\hline FASE & VARIABLE & MEDICIÓN \\
\hline \multirow{3}{*}{ Diseño estratégico } & Alta dirección & $\begin{array}{c}\text { Total Objetivos corporativos / Objetivos } \\
\text { corporativos orientados a innovación. }\end{array}$ \\
\cline { 2 - 3 } & Recursos. & $\begin{array}{c}\text { 1)Total de ingreso/ } \\
\text { Recurso económico autorizado para } \\
\text { innovación }\end{array}$ \\
& $\begin{array}{c}\text { 2)Total horas de trabajo / Tiempo } \\
\text { autorizado para innovación }\end{array}$ \\
\cline { 2 - 3 } & $\begin{array}{c}\text { Búsqueda e identificación } \\
\text { del recurso humano para } \\
\text { la innovación }\end{array}$ & \% de cumplimiento de perfil requerido. \\
\hline
\end{tabular}

Fuente: Elaboración propia.

Tabla 5. Variables de metodología para el mejoramiento de la innovación empresarial en la fase Procesos

\begin{tabular}{|c|c|c|}
\hline FASE & VARIABLES & DESCRIPCIÓN \\
\hline \multirow{4}{*}{ Proceso } & Herramientas & $\begin{array}{l}\text { Reúne equipos y tecnologías, encuestas, departamento de } \\
\text { I+D+i, sistemas de gestión para hacer la gestión de la } \\
\text { innovación. Para innovar se requiere estar organizados por lo } \\
\text { tanto es importante la existencia de sistemas de gestión que } \\
\text { garanticen la organización de la empresa. }\end{array}$ \\
\hline & $\begin{array}{l}\text { Fuentes internas } \\
\text { y externas de } \\
\text { innovación }\end{array}$ & $\begin{array}{l}\text { Participación de todo el cuerpo de empleados, proveedores, } \\
\text { clientes, autoridades portuarias, aduaneras y ambientales, } \\
\text { universidades, competencia, congresos, sociedad aledaña a la } \\
\text { empresa. Es decir todas partes de donde se puede obtener } \\
\text { información necesaria para la generación de innovación. En esta } \\
\text { variable también se involucran los actores institucionales y se } \\
\text { generan alianzas estratégicas con el fin de propiciar un } \\
\text { ecosistema de innovación adecuado y un posible clúster } \\
\text { teniendo en cuenta la existencia de mecanismos ejemplo la } \\
\text { existencia del proyecto Logport. Se busca conocer la percepción } \\
\text { de la comunidad aledaña a la compañía debido a que la } \\
\text { empresa debe tener responsabilidad social, el crecimiento no } \\
\text { solo se concibe para la empresa, también se considera tener } \\
\text { incidencia en el crecimiento del territorio donde está ubicada y } \\
\text { planes regionales. }\end{array}$ \\
\hline & $\begin{array}{ll}\text { Gestión } & \text { de } \\
\text { innovación en } & \text { en procesos }\end{array}$ & $\begin{array}{l}\text { Gestión comercial, operativa y administrativa. Inclusión de la } \\
\text { innovación en los principales procesos involucrados en la } \\
\text { actividad económica de la empresa. }\end{array}$ \\
\hline & $\begin{array}{l}\text { Plan de } \\
\text { actividades para } \\
\text { la innovación }\end{array}$ & $\begin{array}{l}\text { Reuniones, difusión de la información, brainstorming, } \\
\text { actividades de esparcimiento y recreación que regalan un } \\
\text { ambiente propicio para la generación de ideas, motivación } \\
\text { económica y verbal a los empleados, gestión y participación en } \\
\text { ferias de innovación, proyectos de innovación y capacitaciones } \\
\text { para innovación, publicidad de los nuevos servicios. }\end{array}$ \\
\hline
\end{tabular}

Fuente: Elaboración propia. 
Tabla 6. Medición de variables de metodología para el mejoramiento de la innovación

\begin{tabular}{|c|c|c|}
\hline FASE & VARIABLE & MEDICIÓN \\
\hline \multirow{4}{*}{ Proceso } & Herramientas & $\begin{array}{l}\text { 1) Existencia de sistema de gestión } \\
\text { 2)Total de recursos tecnológicos autorizados para la } \\
\text { gestión de innovación /recursos utilizados }\end{array}$ \\
\hline & $\begin{array}{l}\text { Fuentes internas y } \\
\text { externas de innovación }\end{array}$ & $\begin{array}{l}\text { 1)Total de fuentes internas de información para la } \\
\text { innovación / } \\
\text { Total, de fuentes internas que participan activamente } \\
\text { en pro a la innovación. } \\
\text { 2) Total de fuentes externas de información para la } \\
\text { innovación / } \\
\text { Total, de fuentes externas que participan activamente } \\
\text { en pro a la innovación. }\end{array}$ \\
\hline & $\begin{array}{l}\text { Gestión de innovación } \\
\text { en los procesos }\end{array}$ & $\begin{array}{l}\text { Total, de procesos principales / total de procesos } \\
\text { principales que incluyen innovación }\end{array}$ \\
\hline & $\begin{array}{l}\text { Plan de actividades para } \\
\text { la innovación }\end{array}$ & $\begin{array}{l}\text { Total, de actividades planeadas para la innovación / } \\
\text { Total de actividades ejecutadas para la innovación }\end{array}$ \\
\hline
\end{tabular}

Fuente: Elaboración propia.

Tabla 7. Variables de metodología para el mejoramiento de la innovación empresarial en la fase Resultados

\begin{tabular}{|c|c|c|}
\hline FASE & VARIABLES & DESCRIPCIÓN \\
\hline \multirow[b]{3}{*}{ Resultados } & $\begin{array}{l}\text { Modelo o } \\
\text { prototipo }\end{array}$ & $\begin{array}{l}\text { Diseño del proceso de innovación en la empresa, de esta manera } \\
\text { será más fácil la dinámica de la innovación, se debe difundir a } \\
\text { todos los involucrados. }\end{array}$ \\
\hline & $\begin{array}{c}\text { Sistemas } \\
\text { contables } \\
\text { adecuados }\end{array}$ & $\begin{array}{l}\text { Como resultado de la innovación se encuentra un sistema } \\
\text { contable adecuado y adaptado a la gestión de la innovación, que } \\
\text { permita identificar los activos inmateriales generados por la } \\
\text { innovación, el crecimiento de las ventas como resultado de la } \\
\text { gestión de la innovación y el mejoramiento de servicios } \\
\text { prestados, innovación en términos de beneficio económico. }\end{array}$ \\
\hline & Eficiencia & $\begin{array}{l}\text { Menores costos en operaciones de servicio prestado, nuevos } \\
\text { procesos administrativos que permitan una ágil prestación del } \\
\text { servicio, número de reconocimientos por entidades de comercio } \\
\text { exterior ejemplo (reconocimientos que otorga Proexport, cámara } \\
\text { de comercio) }\end{array}$ \\
\hline Resultados & Cultura & $\begin{array}{l}\text { Cambio en el comportamiento de los empleados orientado a la } \\
\text { innovación, costumbres, motivación permanente, } \\
\text { experimentación, ruptura de status quo, frecuencia de reuniones, } \\
\text { apropiación del conocimiento para la innovación. Debe haber } \\
\text { transferencia de conocimienton, captura y apropiación de nuevo } \\
\text { conocimiento, en esta parte la innovación hace parte del ADN } \\
\text { de la organización. Y los resultados obtenidos se difunden con } \\
\text { el resto de la organización. }\end{array}$ \\
\hline
\end{tabular}

Fuente: Elaboración propia 
Tabla 8. Medición de variables de metodología para el mejoramiento de la innovación empresarial en la fase Resultados

\begin{tabular}{|c|l|l|}
\hline FASE & \multicolumn{1}{|c|}{ VARIABLE } & \multicolumn{1}{c|}{ MEDICIÓN } \\
\hline \multirow{5}{*}{ Resultados } & Modelo o prototipo & $\begin{array}{l}\text { No. de procesos mejorados / No. de actividades de } \\
\text { innovación. }\end{array}$ \\
\cline { 2 - 4 } & $\begin{array}{l}\text { Sistemas contables } \\
\text { adecuados }\end{array}$ & $\begin{array}{l}\text { 1)Total inversión para la innovación / Total ventas } \\
\text { facturadas resultado de las actividades de innovación } \\
\text { 2) Total de activos inmateriales / total de activos } \\
\text { inmateriales resultado de actividades de innovación }\end{array}$ \\
\cline { 2 - 4 } & Eficiencia & $\begin{array}{l}\text { Costos de operación por prestación del servicio previo a } \\
\text { implementación de actividades de innovación - Costos de } \\
\text { operación por prestación del servicio posterior a } \\
\text { implementación de actividades de innovación }\end{array}$ \\
\cline { 2 - 4 } & $\begin{array}{l}\text { Nivel 1. Improvisación. } \\
\text { Nivel 2. Lista de Proyectos. } \\
\text { Nivel 3. Portafolio de proyectos. } \\
\text { Nivel 4. Organización para innovar. } \\
\text { Nivel 5. Cultura de la innovación. }\end{array}$ \\
\hline
\end{tabular}

Fuente: Elaboración propia

\section{CONCLUSIONES}

La presente investigación mostró el estado actual de innovación organizacional de cinco empresas del sector logístico en la ciudad de Cartagena, evidenciando que ninguna de éstas ha podido alcanzar un estado idóneo de innovación organizacional. Lo anterior, debido a que no cumplen integralmente con los nueve ejes utilizados para medir la innovación en estas empresas (cultura, estrategia, planificación, personas/medios, procesos, herramientas, producto/servicio, eficiencia y capitalización).

En promedio, las empresas analizadas alcanzan los niveles de innovación requeridos (más del 80\%) en cinco de nueve ejes medidos, lo cual muestra la necesidad que tienen las empresas del sector logístico de mejorar sus niveles de innovación organizacional con el objetivo de alcanzar el estado ideal (100\% en los nueve ejes). Con base en lo anterior, se estructura la propuesta metodológica para el mejoramiento de la innovación organizacional, la cual se describe en la segunda sección de los resultados.

La propuesta metodológica se realizó teniendo en cuenta la importancia que tuvo el eje de planificación en las empresas analizadas, razón por la cual, la primera fase de la propuesta metodológica se enfoca en la planificación o direccionamiento estratégico. Adicionalmente, la propuesta tiene en cuenta la interdependencia que mostraron los ejes de procesos y herramientas, los cuales deben desarrollarse conjuntamente para dar mejores resultados. Finalmente, se propende a la creación de una cultura innovadora en la empresa, ya que las 
empresas serán innovadoras en la medida en que se interiorice una cultura organizacional enfocada en este sentido.

Pese a todos los esfuerzos que se están realizando en las empresas del sector logístico para comercio exterior de Cartagena de Indias, se evidencia que aún tienen algunas debilidades para la gestión y generación de innovación, razón por lo cual se realizó esta propuesta metodológica para el mejoramiento de la innovación empresarial en el sector. Como recomendación final, se resalta que es necesario gestionar espacios y programas de innovación dentro de las empresas del sector logístico para comercio exterior de la ciudad de Cartagena, debido a que actualmente existen muchos vacíos conceptuales en las empresas, las cuales se encuentran inmersas en problemas para el desarrollo de sus procesos administrativos..

\section{REFERENCIAS}

Acevedo, A. y Linares, C. (2008). El proceso de innovación dentro del diseño estratégico de las organizaciones. Revista de la facultad de ingeniería industrial, 11(2), 33-44. Recuperado de https://www.redalyc.org/pdf/816/81619829005.pdf

Afuach, A. (1999). La dinámica de la innovación organizacional: un nuevo enfoque para lograr ventaja competitiva. México D.F: OXFORD University Press.

Albornoz, M. (2009). Indicadores de Innovacion: Las dificultades de un concepto en evolución. CTS, 13(5) , 9-25. Recuperado de https://www.researchgate.net/publication/41150842_Indicadores_de_innovacion_las _dificultades_de_un_concepto_en_evolucion

Arraut, L. (2008). La innovacion de tipo organizacional en las empresas manufactureras de cartagena de Indias. Semestre economico, Universidad de Medellin. 11 (22), 185203. Recuperado de http://www.scielo.org.co/pdf/seec/v11n22/v11n22a9.pdf

Arraut, L. (2010). La gestión de la calidad como innovación organizacional para la productividad en la empresa. Revista EAN, 69, 20-41. Recuperado de https://www.redalyc.org/pdf/206/20619966002.pdf

Bonache Perez, J. (1999). El estudio de casos como estrategia de construcción teórica: características, críticas y defensas. Cuadernos de Economía y Dirección de la Empresa, 3, 123-140. Recuperado de https://dialnet.unirioja.es/servlet/articulo?codigo=195459

Bustamante, A., Dávila, G. y Castañón, R. (2019). Gestión de la tecnología e innovación: un modelo de Redes Bayesianas. Economía: Teoría y Práctica, 50, 63-100. doi https://doi.org/10.24275/etypuam/ne/502019/teran

Camara de Comercio de Cartagena (2014). Comportamiento del Comercio Exterior De Bolivar Informe I Semestre 2014. Seguimiento al TLC Con Estados Unidos. Cartagena: 
Camara de Comercio de Cartagena ISSN. 1909-9800 pag. 1-35. Recuperado de http://www2.cccartagena.org.co/sites/default/files/2012101850_inv_comercio_exteri or_i_semestre_2014.pdf

Comisión Europea (1995). Libro Verde de la Innovación. Bruselas: Comisión Europea. Recuperado de https://eur-lex.europa.eu/resource.html?uri=cellar:eb5dae41-104d4724-ac99-d7cbcfa11b86.0008.01/DOC_1\&format=PDF

COTEC, F. (2007). Innovación en el sector hotelero. Madrid: COTEC, Fundación para la innovación tecnológica. Recuperado de https://www.ithotelero.com/wpcontent/uploads/2013/08/proyecto13-cotec.pdf

COTEC, F. (2013). La innovación en las Pyme españolas. Madrid: COTEC, Fundacion para la Innovacion Tecnologica. Recuperado de http://informecotec.es/media/46_Inno_Pymes_Esp.pdf

D’alvano, L. (2011). Uso de técnicas y el desarrollo del proceso de innovación en las organizaciones de servicio. Instituto de estudios superiores de Administración IESA. Recuperado de http://oa.upm.es/11543/1/INVE_MEM_2011_105551.pdf

Drucker, P. (1998). The Discipline of innovation. Harvard Business review. Reprint 98604, $1-8$.

Dubrova, T., Ermolina, A. y Esenin, M. (2019). Innovative activities of SMEs in Russia: Constraints and growth factors. International journal of Economics and Business administration, 7, 26-40. Recuperado de https://ideas.repec.org/a/ers/ijebaa/vviiy2019ispecial2p26-40.html

Garud, R., Tuertscher, P. y Van de Ven, A. Perspectives on innovation processes. The academy of management annals, 7(1), 773-817. doi 10.1080/19416520.2013.791066

Hamel, G. (2001). Liderando la revolución. Bogotá: Ediciones Norma.

Hernandez, R. (2017). Metodología de la investigación. México D.F: Mc Graw Hill. Recuperado de https://www.uca.ac.cr/wpcontent/uploads/2017/10/Investigacion.pdf

Kita, P. y Grossmanová, M. (2014). Reflection of Bratislava retail network in selected aspects of consumer behaviour. Business: theory and practice, 15(3), 279-284. doi 10.3846/btp.2014.28

Lam, A. (2010). Organizaciones innovadoras: estructura, aprendizaje y adaptación. BBVA, 163-177. Recuperado de https://www.bbvaopenmind.com/articulos/organizacionesinnovadoras-estructura-aprendizaje-y-adaptacion/

Mauri, J. y Canós, L. (2004). Optimización de la estructura organizativa basada en el modelo de la gestión por procesos, de una PYME del sector logístico. VIII Congreso de la Ingeniería de la Organización, 9 y 10 de septiembre, Leganés, España. 
Recuperado de http://www.adingor.es/congresos/web/uploads/cio/cio2004/829838.pdf

McDonough, E., Zack, M., Lin, H.-E., y Iris, B. (2008). Integrating Innovation style and Knowledge Into Strategy. MITSloan Management Review, 50(1), 53-58. Recuperado de

https://www.researchgate.net/publication/263962893_Integrating_Innovation_Style _and_Knowledge_Into_Strategy_If_you_devise_strategy

OECD (2005). Manual de Oslo: guía para la recogida e interpretación de datos sobre innovación, 3a ed. París: OECD Publications. Recuperado de http://www.itq.edu.mx/convocatorias/manualdeoslo.pdf

Pertuz, V. y Pérez, A. (2016). Modelo de cultura organizacional innovadora en caficultores del departamento del Cesar, Colombia. Revista de investigación desarrollo e innovación, 6(2), 117-130 doi: http://dx.doi.org/10.19053/20278306.3687

RICYT -Red Iberoamericana de Ciencia y Tecnología- (2001). Manual de Bogotá: Normalización de Indicadores de Innovación Tecnológica en América Latina y el Caribe. Red Iberoamericana de Ciencia y Tecnología (RICYT), Organización de Estados Americanos (OEA), Programa CYTED. Recuperado de https://www.academia.edu/701749/Normalizaci\%C3\%B3n_de_indicadores_de_inno vaci\%C3\%B3n_tecnol\%C3\%B3gica_en_Am\%C3\%A9rica_latina_y_el_Caribe_manual_ de_Bogot\%C3\%A1

Sawhney, M., Wolcott, R., y Arroniz, I. (2006). The 12 Different Ways for Companies to Innovate. MITSloan Management Review 47 (3), 74-81. Recuperado de http://marketing.mitsmr.com/offers/BX/47314-The-12-Different-Ways-for-

Companies-to-Innovate.pdf

Varela, R. (2001). Innovación empresarial: arte y ciencia en la creación de empresas. Bogota: Pearson educacion de Colombia. ISBN: 958-699-023-0. Recuperado de https://www.icesi.edu.co/unicesi/todas-las-noticias/1757-innovacion-empresarialarte-y-ciencia-en-la-creacion-de-empresa

Yin, R. (1994). Case study research: design and methods. London: Sage Publications. Recuperado de https://books.google.com.co/books/about/Case_Study_Research.html?hl=es\&id=F zawlAdilHkC\&redir_esc=y

Yrigoyen, J. (2013). Explorando distintos Tipos de Innovacion en Micro y Pequeñas empresas Peruanas. Journal of Technology Management \& Innovation. Vol. 8 special issue Altec ISSN: 0718-2724, 72- 82. Recuperado de https://scielo.conicyt.cl/pdf/jotmi/v8s1/art62.pdf 